\title{
Genetic Divergence of Maize Genotypes under Normal and Water Stress Conditions
}

\author{
K. Vijay Kumar ${ }^{1 *}$, K. V. Radha Krishna ${ }^{2}$, T. Pradeep ${ }^{3}$, \\ D. Vishnuvardhan Reddy ${ }^{3}$ and M. H. V. Bhave ${ }^{4}$ \\ ${ }^{1}$ Agricultural Research Station, PJTSAU, Mudhol, India \\ ${ }^{2}$ Department of Genetics \& Plant Breeding, College of Agriculture, PJTSAU), Rajendranagar, \\ Hyderabad-500 030, India \\ ${ }^{3}$ PJTSAU, Rajendranagar, Hyderabad, India \\ ${ }^{4}$ Department of Mathematics \& Statistics, College of Agriculture, PJTSAU, \\ Rajendranagar, Hyderabad, India \\ *Corresponding author
}

\begin{abstract}
A B S T R A C T
Seventy five maize genotypes were evaluated to study the genetic divergence under two drought stress conditions i.e. moderate stress and severe stress along with well watered

Keywords

Maize, genetic diversity, cluster analysis, drought stress

Article Info

Accepted:

xx May 2018

Available Online:

xx June 2018

conditions. The experiment was carried out at Maize Research Centre, Rajendranagar, Hyderabad, during rabi 2011-12. The seventy five genotypes were grouped into nine distinct clusters. The cluster VII, cluster VIII and cluster VII were the largest and lowest genotypes were included in cluster II, cluster V and clusters V and IX under normal, moderate drought stress and severe drought stress conditions, respectively. The highest inter-cluster distance was observed between, cluster IV and VIII, V and VII and III and V under normal, moderate drought stress and severe drought stress conditions, respectively. Chlorophyll fluorescence and ear weight showed maximum contribution towards total divergence among different characters studied, in all the three water regimes. The cluster IV, cluster V and cluster III showed high mean values for plant height, ear length, ear weight, harvest index and grain yield under normal, moderate drought stress and severe drought stress conditions, respectively. The genotypes with wide genetic diversity can be utilized in future breeding programme to evolve drought tolerant hybrids to mitigate the adverse effects of climate change.
\end{abstract}

\section{Introduction}

In India Maize (Zea mays L.) has become an important cereal crop after rice \& wheat. It is grown in an area of 8.80 million hectares with production of 22.56 million tonnes and productivity of $2680 \mathrm{~kg} / \mathrm{ha}$ (Centre for Monitoring Indian Economy, 2016). In recent years, the productivity of maize was not increased significantly (2007-08: $2335 \mathrm{~kg} / \mathrm{ha}$ to 2015-16: $2562 \mathrm{~kg} / \mathrm{ha}$ ), even though $90 \%$ of the cultivated area is under high yielding single cross hybrids. The low grain yields can be attributed to a number of constraints which include biotic stress such as diseases, pests and parasitic weeds and abiotic stresses such as low soil fertility and drought. Among them, drought stress or water deficit is an envitable and recurring feature of World Agriculture. It has been estimated that about one third of the world's potentially arable land suffers water shortage and crop yields are often reduced by 
drought (Khan et al., 2004). The development of crops with increased tolerance to abiotic stresses especially drought and salinity is much required throughout the world (Denby and Gehring, 2005). Drought remains the most important devastating factor in Maize and it has different effects on the crop depending on the growth stage at which it occurs (Umar et al., 2015). Soil moisture deficit in maize may cause drastic yield reduction, especially if it occurs during the reproductive phase (NeSmith and Ritchie, 1992; Basseti and Westgate, 1993).

Banziger (2000) observed that the full sib family selection was the most extensively used at CIMMYT to improve maize populations for drought and low-N tolerance. Precise selection of elite genotypes is very important to breed for any specific area (Ashofteh Beiragi et al., 2010). Several studies on maize have shown that inbred lines from diverse stocks were more productive than crosses of inbred lines from the same variety (Vasal, 1998). Saxena et al., (1998) also reported that manifestation of heterosis usually depends on the genetic divergence of the two parental lines. Multivariate analysis based on Mahalonobis $\mathrm{D}^{2}$ statistic is widely used for effective discrimination of genotypes on the basis of genetic diversity (Murthy and Arunachalam, 1966). Several studies were published on classification of germplasm collections and classification of maize inbreds with comparision of cluster analysis algorithm. The present study was undertaken to evaluate the genetic diversity among the maize genotypes specially for drought tolerance which can be exploited in future maize breeding programmes.

\section{Materials and Methods}

A total of seventy five maize genotypes obtained, of which 42 from Maize Research Centre, Rajendranagar, Hyderabad and 33 from Agricultural Research Station, Karimnagar were included in the present investigation. The experimental material was evaluated under three conditions; one normal (irrigated) and two drought stress conditions viz., moderate drought stress conditions (suspending the irrigation 20 days before flowering and during grain filling stage) and severe drought stress conditions (suspending the irrigation 40 days after sowing). For each condition, the experimental material was sown in randomized block design with two replications. Replication wise, each genotype was sown in a row of $4 \mathrm{~m}$ length spaced $75 \mathrm{~cm}$ apart with a $20 \mathrm{~cm}$ distance between plants with in the row. All the recommended agronomic practices and plant protection measures were taken up in time to raise the crop.

Data was recorded on seventeen morpho physiological and yield attributing characters. Observations were recorded on no.of leaves per plant, plant height $(\mathrm{cm})$, ear height $(\mathrm{cm})$, ear length $(\mathrm{cm})$, ear girth $(\mathrm{cm})$, no.of kernel rows per ear, no.of kernels per row, 100 kernel weight $(\mathrm{g})$, ear weight $(\mathrm{g})$, Harvest Index (\%) and grain yield per plant (g) on five randomly selected plants for each entry in each replication.

Data pertaining to days to $50 \%$ tasseling, days to $50 \%$ silking and Anthesis-Silking Interval and leaf firing score were recorded on plot basis. The SPAD chlorophyll meter reading and Chlorophyll flourometer readings for leaf chlorophyll content and chlorophyll fluorescence respectively were taken at 90 days after sowing on top third leaf of randomly selected five plants. Mean values were subjected to statistical analysis.

Genetic divergence was estimated as per Mahalanobis D ${ }^{2}$ statistic (1936) and clustering of genotypes was done according to Tocher's method as described by Rao (1952). 


\section{Results and Discussion}

The analysis of variance revealed highly significant differences among the genotypes for all the studied characters under all three conditions indicating the existence of genetic variability among the experimental material in all the tested water regimes. All the 75 genotypes were grouped into nine clusters each in each condition (Table 1). Clustering pattern of genotypes clearly indicated that majority of the inbred lines showed considerable genetic diversity by occupying different clusters in each condition. A similar study was carried out by Chen FaBo et al., (2007) who grouped 186 genotypes into ten clusters. In normal condition, it was revealed that cluster VII comprised of maximum number (21) of genotypes followed by cluster I and cluster VI with 12 and 9 genotypes, respectively. The lowest (3) number of genotypes were included in cluster II. Whereas under moderate stress condition, cluster VIII was the largest comprised of 18 genotypes followed by clusters I and IX with 14 genotypes. Single genotype was included in the cluster $\mathrm{V}$. The maximum number of genotypes was observed in clusters I and VIII with 15 genotypes followed by cluster VI with 12 genotypes and the lowest (3) was reported in clusters V and IX in case of severe stress condition.

The average intra and inter cluster distance values were worked out from divergence analysis for each character (Table 2). The intra cluster distances were lower than the inter cluster distances indicating heterogeneous and homogeneous nature between and within the groups, respectively. The maximum intra cluster distance was observed by cluster VIII under both normal and severe stress conditions and cluster VI in moderate stress condition. Cluster $\mathrm{V}$ in moderate stress condition had only a single genotype, hence minimum intra cluster distance was observed.
The hybrids are expected to manifest maximum heterosis by involving the parents from most divergent clusters which generates wide variability. This was supported by appreciable amount of variation observed for the cluster means under all the tested conditions.

The highest inter cluster distance was observed between clusters IV and VIII (221.29) followed by clusters III and VIII (210.77) under normal conditions. Whereas, the highest distance was obtained by clusters V and VII (237.93) followed by clusters IV and VII (198.41) and clusters V and VI (194.47) in case of moderate stress conditions, while in case of severe stress conditions highest distance was obtained by clusters III and V (164.29), followed by II and V (148.82) and $\mathrm{I}$ and $\mathrm{V}$ (139.87). This suggests more genetic variability in the genetic architecture of the genotypes included in these clusters under each situation. The genotypes belong to the cluster separated by high statistical distance could be utilized as parents in hybridization programme for yield improvement.

The results on the contribution of individual characters towards the total divergence under normal and drought stressed conditions are presented in Table 3. Of the seventeen characters studied, the percent contribution towards total divergence was the highest for chlorophyll fluorescence followed by ear weight in all three water regimes.

Mean values of clusters for yield and its contributing characters and physiological parameters are presented in the Table 3. It shows that the genotypes with high values of plant height, ear length, ear weight, harvest index and grain yield were included in cluster IV, cluster V and cluster III under normal, moderate and severe stress conditions, respectively. 
Table.1 Grouping of 75 maize genotypes into clusters under normal, moderate stress and severe stress conditions

\begin{tabular}{|c|c|c|c|}
\hline Cluster & Condition & No. of genotypes & Genotypes \\
\hline \multirow[t]{3}{*}{ Cluster I } & $\mathrm{N}$ & 12 & $\begin{array}{l}\text { BMLDT-2, BMLDT-64, BMLDT-97, BMLDT-87, BMLDT-16, BMLDT-56, BMLDT-86, BMLDT-27, BMLDT-29, BMLDT-95, BMLDT-134 } \\
\text { and BMLDT-38 }\end{array}$ \\
\hline & MS & 14 & $\begin{array}{l}\text { BMLDT-1, BMLDT-87, BMLDT-5, BMLDT-11, BMLDT-56, BMLDT-2, BMLDT-24, BMLDT-13, BMLDT-88, BMLDT-129, BMLDT-135, } \\
\text { BMLDT-97, BMLDT-134, } 1063\end{array}$ \\
\hline & SS & 15 & $\begin{array}{l}\text { BMLDT-2, BMLDT-87, BMLDT-1, BMLDT-129, BMLDT-134, BMLDT-56, BMLDT-5, BMLDT-124, BMLDT-11, BMLDT-24, BMLDT-135, } \\
\text { BMLDT-97, BMLDT-4, BMLDT-14, } 1063\end{array}$ \\
\hline \multirow[t]{3}{*}{ Cluster II } & $\mathrm{N}$ & 3 & BMLDT-132, BMLDT-133 and BMLDT-131 \\
\hline & MS & 3 & BMLDT-95, BMLDT-103, BMLDT-82 2 2 \\
\hline & SS & 8 & BMLDT-9, BMLDT-99, BMLDT-38, BMLDT-88, BMLDT-21, BMLDT-34, BMLDT-91 and BMLDT-86. \\
\hline \multirow[t]{3}{*}{ Cluster III } & $\mathrm{N}$ & 8 & BMLDT-4, BMLDT-45, BMLDT-11, 1063, BMLDT-24, BMLDT-99, BMLDT-124, BMLDT-135 \\
\hline & MS & 5 & BMLDT-9, BMLDT-34, BMLDT-99, BMLDT-124, BMLDT-86 \\
\hline & SS & 4 & BMLDT-15, BMLDT-27, BMLDT-29 and BMLDT-45 \\
\hline \multirow[t]{3}{*}{ Cluster IV } & $\mathrm{N}$ & 4 & BMLDT-5, BMLDT-21, BMLDT-15 and BMLDT-91 \\
\hline & MS & 7 & BMLDT-15, BMLDT-21, BMLDT-27, BMLDT-29, BMLDT-38, BMLDT-45, BMLDT-4 \\
\hline & SS & 11 & $\begin{array}{l}\text { BMLDT-3, BMLDT-17, BMLDT-8, BMLDT-106, BMLDT-7, BMLDT-12, BMLDT-10, BMLDT-110, BMLDT-109, BMLDT-119 and BMLDT- } \\
108\end{array}$ \\
\hline \multirow[t]{3}{*}{ Cluster V } & $\mathrm{N}$ & 5 & BMLDT-9, BMLDT-13, BMLDT-129, BMLDT-39 and BMLDT-78 \\
\hline & MS & 1 & BMLDT-91 \\
\hline & SS & 3 & BMLDT-101, BMLDT-102 and BMLDT-107 \\
\hline \multirow[t]{3}{*}{ Cluster VI } & $\mathrm{N}$ & 9 & BMLDT-14, BMLDT-88, BMLDT-25, BMLDT-34, BMLDT-18, BMLDT-80, BMLDT-1, BMLDT-36, and BMLDT-37 \\
\hline & MS & 11 & BMLDT-3, BMLDT-8, BMLDT-108, BMLDT-109, BMLDT-119 BMLDT-10, BMLDT-3, BMLDT-12, BMLDT-17, BMLDT-101, BMLDT-106 \\
\hline & SS & 12 & $\begin{array}{l}\text { BMLDT-16, BMLDT-40, BMLDT-13, BMLDT-25, BMLDT-78 BMLDT-32, BMLDT-112, BMLDT-80, BMLDT-118, BMLDT-37, BMLDT-74 } \\
\text { and BMLDT-39. }\end{array}$ \\
\hline \multirow[t]{3}{*}{ Cluster VII } & $\mathrm{N}$ & 21 & $\begin{array}{l}\text { BMLDT-20, BMLDT-103, BMLDT-110, BMLDT-114, BMLDT-57, BMLDT-23, BMLDT-118, BMLDT-32, BMLDT-58, BMLDT-100, } \\
\text { BMLDT-112, BMLDT-33, BMLDT-82, BMLDT-40, BMLDT-70, BMLDT-74, BMLDT-26,, BMLDT-6, BMLDT-73, BMLDT-113 and } \\
\text { BMLDT-53 }\end{array}$ \\
\hline & MS & 2 & 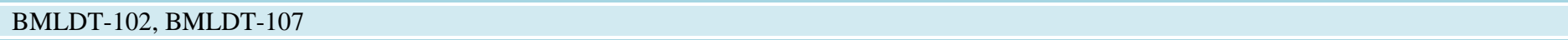 \\
\hline & SS & 15 & $\begin{array}{l}\text { BMLDT-20, BMLDT-23, BMLDT-18, BMLDT-26, BMLDT-36, BMLDT-57, BMLDT-132, BMLDT-53, BMLDT-73, BMLDT-58, BMLDT-70, } \\
\text { BMLDT-33, BMLDT-100, BMLDT-113, BMLDT-6, }\end{array}$ \\
\hline \multirow[t]{3}{*}{ Cluster VIII } & $\mathrm{N}$ & 7 & BMLDT-3, BMLDT-12, BMLDT-108, BMLDT-101, BMLDT-106, BMLDT-102 AND BMLDT-107 \\
\hline & MS & 18 & $\begin{array}{l}\text { BMLDT-6, BMLDT-18, BMLDT-132, BMLDT-133,BMLDT-131, BMLDT-32 BMLDT-53, BMLDT-100, BMLDT-113, BMLDT-78 BMLDT- } \\
\text { 112, BMLDT-20,BMLDT-23, BMLDT-57, BMLDT-58, BMLDT-80, BMLDT-33 and BMLDT-110 }\end{array}$ \\
\hline & SS & 4 & BMLDT-64, BMLDT-114, BMLDT-131 and BMLDT-133 \\
\hline \multirow[t]{3}{*}{ Cluster IX } & $\mathrm{N}$ & 6 & BMLDT-10, BMLDT-17, BMLDT-8, BMLDT-109, BMLDT-119 and BMLDT-3, \\
\hline & MS & 14 & $\begin{array}{l}\text { BMLDT-14, BMLDT-40, BMLDT-16, BMLDT-25, BMLDT-64, BMLDT-26, BMLDT-36, BMLDT-37, BMLDT-74, BMLDT-114, BMLDT-39, } \\
\text { BMLDT-118, BMLDT-70 and BMLDT-73 }\end{array}$ \\
\hline & SS & 3 & BMLDT-95, BMLDT-103 and BMLDT-82 2 \\
\hline
\end{tabular}

Note: N-Normal; MS-Moderate stress; SS-Severe stress. 
Table.2 Average inter and intra cluster distances $\left(D=\sqrt{ } D^{2}\right)$ values under normal, moderate stress and severe stress conditions

\begin{tabular}{|c|c|c|c|c|c|c|c|c|c|c|}
\hline Cluster & Condition & Cluster I & Cluster II & Cluster III & Cluster IV & Cluster V & Cluster VI & Cluster VII & Cluster VIII & Cluster IX \\
\hline \multirow[t]{3}{*}{ Cluster I } & $\mathrm{N}$ & 56.08 & 77.13 & 78.39 & 89.05 & 87.28 & 74.00 & 99.41 & 198.12 & 124.29 \\
\hline & MS & 23.91 & 35.59 & 39.45 & 64.86 & 101.70 & 113.35 & 159.00 & 68.53 & 54.67 \\
\hline & SS & 30.85 & 45.13 & 69.42 & 105.17 & 139.87 & 58.28 & 70.92 & 62.55 & 43.21 \\
\hline \multirow[t]{3}{*}{ Cluster II } & $\mathrm{N}$ & & 37.95 & 106.39 & 123.03 & 98.84 & 68.33 & 81.05 & 159.51 & 86.62 \\
\hline & MS & & 20.48 & 59.88 & 85.93 & 125.45 & 82.16 & 126.83 & 42.30 & 39.48 \\
\hline & SS & & 32.95 & 44.89 & 118.35 & 148.82 & 85.16 & 96.05 & 88.19 & 60.32 \\
\hline \multirow[t]{3}{*}{ Cluster III } & $\mathrm{N}$ & & & 60.03 & 106.39 & 74.13 & 82.03 & 98.50 & 210.77 & 139.59 \\
\hline & MS & & & 24.47 & 39.12 & 72.11 & 132.99 & 127.88 & 95.25 & 83.87 \\
\hline & SS & & & 24.74 & 137.70 & 164.29 & 114.68 & 123.85 & 111.90 & 85.51 \\
\hline \multirow[t]{3}{*}{ Cluster IV } & $\mathrm{N}$ & & & & 45.14 & 111.28 & 121.46 & 152.18 & 221.29 & 156.31 \\
\hline & MS & & & & 25.56 & 46.95 & 154.18 & 198.41 & 121.74 & 111.59 \\
\hline & SS & & & & 32.97 & 48.72 & 82.42 & 65.19 & 80.38 & 73.54 \\
\hline \multirow[t]{3}{*}{ Cluster V } & $\mathrm{N}$ & & & & & 47.11 & 63.02 & 79.60 & 168.19 & 103.97 \\
\hline & MS & & & & & 0.00 & 194.47 & 237.93 & 161.81 & 150.13 \\
\hline & SS & & & & & 26.38 & 118.01 & 99.45 & 114.54 & 107.18 \\
\hline \multirow[t]{3}{*}{ Cluster VI } & $\mathrm{N}$ & & & & & & 45.77 & 58.37 & 159.59 & 87.29 \\
\hline & MS & & & & & & 31.70 & 54.62 & 62.82 & 85.41 \\
\hline & SS & & & & & & 26.84 & 33.07 & 42.67 & 43.54 \\
\hline \multirow[t]{3}{*}{ Cluster VII } & $\mathrm{N}$ & & & & & & & 53.04 & 156.07 & 90.62 \\
\hline & MS & & & & & & & 26.60 & 103.57 & 127.63 \\
\hline & SS & & & & & & & 23.57 & 40.81 & 43.54 \\
\hline \multirow{3}{*}{$\begin{array}{c}\text { Cluster } \\
\text { VIII }\end{array}$} & $\mathrm{N}$ & & & & & & & & 71.70 & 101.10 \\
\hline & MS & & & & & & & & 22.75 & 32.94 \\
\hline & SS & & & & & & & & 35.21 & 47.42 \\
\hline \multirow[t]{3}{*}{ Cluster IX } & $\mathrm{N}$ & & & & & & & & & 54.76 \\
\hline & MS & & & & & & & & & 22.06 \\
\hline & SS & & & & & & & & & 27.52 \\
\hline
\end{tabular}

Note: Figures in parenthesis indicate D values; Diagonal values indicate intra-cluster distance.

N-Normal; MS-Moderate stress; SS-Severe stress. 
Table.3 Cluster means for morpho physiological and yield contributing characters under normal, moderate stress and severe stress conditions

\begin{tabular}{|c|c|c|c|c|c|c|c|c|c|c|c|c|c|c|c|}
\hline \multirow[t]{2}{*}{ Cluster } & \multicolumn{3}{|c|}{ No.of leaves/plant } & \multicolumn{3}{|c|}{$\begin{array}{c}\text { Days to } \mathbf{5 0 \%} \\
\text { tasseling }\end{array}$} & \multicolumn{3}{|c|}{ Days to $50 \%$ silking } & \multicolumn{3}{|c|}{ ASI } & \multicolumn{3}{|c|}{ Plant height (cm) } \\
\hline & $\mathrm{N}$ & MS & SS & $\mathrm{N}$ & MS & SS & $\mathrm{N}$ & MS & SS & $\mathrm{N}$ & MS & SS & $\mathrm{N}$ & MS & SS \\
\hline $\mathbf{I}$ & 11.97 & 11.12 & 10.04 & 77.71 & 68.07 & 71.03 & 80.71 & 72.96 & 77.90 & 3.08 & 4.96 & 7.00 & 176.42 & 134.19 & 116.79 \\
\hline II & 12.22 & 10.44 & 9.57 & 85.50 & 68.83 & 69.63 & 89.00 & 77.33 & 75.81 & 3.50 & 6.17 & 6.19 & 174.72 & 124.44 & 119.95 \\
\hline III & 11.94 & 11.10 & 10.96 & 83.19 & 66.30 & 68.88 & 86.25 & 72.00 & 74.38 & 3.06 & 5.50 & 5.63 & 147.27 & 134.10 & 146.25 \\
\hline IV & 13.04 & 12.55 & 9.88 & 74.38 & 65.93 & 71.36 & 76.50 & 70.21 & 79.27 & 2.13 & 4.29 & 7.91 & 189.33 & 157.79 & 103.02 \\
\hline $\mathbf{V}$ & 11.43 & 11.84 & 9.78 & 77.90 & 64.00 & 71.67 & 81.10 & 70.50 & 84.17 & 3.20 & 6.50 & 12.50 & 132.15 & 159.17 & 101.67 \\
\hline VI & 11.82 & 10.94 & 9.40 & 78.33 & 67.32 & 72.00 & 81.06 & 73.27 & 81.38 & 2.72 & 5.95 & 9.38 & 147.75 & 118.12 & 97.55 \\
\hline VII & 11.10 & 10.17 & 9.49 & 80.17 & 65.25 & 71.17 & 83.38 & 75.75 & 80.57 & 3.21 & 10.50 & 9.40 & 132.64 & 111.58 & 99.34 \\
\hline VIII & 11.19 & 10.65 & 9.33 & 77.00 & 68.58 & 72.00 & 79.79 & 76.36 & 81.13 & 2.79 & 7.83 & 9.13 & 128.81 & 116.39 & 132.33 \\
\hline IX & 11.78 & 10.68 & 9.22 & 82.08 & 68.04 & 72.00 & 84.75 & 75.11 & 80.00 & 2.67 & 7.07 & 8.00 & 146.94 & 112.02 & 110.16 \\
\hline Mean & 11.83 & 11.05 & 9.74 & 79.58 & 66.92 & 71.08 & 82.50 & 73.72 & 79.40 & 2.93 & 6.53 & 8.35 & 152.89 & 129.76 & 114.12 \\
\hline \multicolumn{16}{|c|}{ Contribution of each character to the divergence } \\
\hline $\begin{array}{l}\text { No. of times } \\
\text { appearing } \\
\text { first in } \\
\text { ranking } \\
\end{array}$ & 0 & 0 & 0 & 32 & 1 & 2 & 0 & 2 & 6 & 0 & 2 & 0 & 796 & 68 & 211 \\
\hline $\begin{array}{l}\% \text { of } \\
\text { contribution } \\
\text { toward total } \\
\text { divergence }\end{array}$ & 0.00 & 0.00 & 0.00 & 1.15 & 0.04 & 0.07 & 0.00 & 0.07 & 0.22 & 0.00 & 0.07 & 0.00 & 28.68 & 2.45 & 7.60 \\
\hline
\end{tabular}




\begin{tabular}{|c|c|c|c|c|c|c|c|c|c|c|c|c|c|c|c|}
\hline \multirow[t]{2}{*}{ Cluster } & \multicolumn{3}{|c|}{ Ear height (cm) } & \multicolumn{3}{|c|}{ Ear length (cm) } & \multicolumn{3}{|c|}{ Ear girth $(\mathrm{cm})$} & \multicolumn{3}{|c|}{ No.of kernel rows/ear } & \multicolumn{3}{|c|}{ No.of kernels/row } \\
\hline & $\mathrm{N}$ & MS & SS & $\mathrm{N}$ & MS & SS & $\mathrm{N}$ & MS & SS & $\mathrm{N}$ & MS & SS & $\mathrm{N}$ & MS & SS \\
\hline $\mathbf{I}$ & 65.64 & 51.73 & 43.47 & 13.00 & 12.64 & 10.82 & 12.47 & 11.15 & 9.71 & 13.91 & 12.37 & 10.53 & 23.10 & 20.35 & 15.96 \\
\hline II & 63.06 & 48.06 & 42.52 & 14.15 & 13.00 & 12.88 & 11.13 & 10.46 & 10.49 & 10.78 & 11.50 & 11.36 & 17.83 & 15.87 & 20.32 \\
\hline III & 52.61 & 48.57 & 58.25 & 12.38 & 14.10 & 13.09 & 11.23 & 11.77 & 11.81 & 12.22 & 12.08 & 12.68 & 19.33 & 21.59 & 24.80 \\
\hline IV & 80.83 & 65.38 & 38.85 & 16.67 & 14.61 & 10.12 & 13.69 & 12.27 & 8.74 & 14.08 & 13.70 & 8.69 & 33.78 & 28.79 & 10.04 \\
\hline $\mathbf{V}$ & 49.42 & 65.17 & 34.83 & 14.03 & 15.71 & 10.52 & 12.22 & 11.70 & 8.61 & 13.51 & 13.20 & 8.87 & 28.18 & 28.00 & 10.30 \\
\hline VI & 54.35 & 46.70 & 34.13 & 11.52 & 11.51 & 8.74 & 11.27 & 9.51 & 8.37 & 12.32 & 10.11 & 9.54 & 20.94 & 13.33 & 11.71 \\
\hline VII & 43.21 & 35.46 & 36.71 & 11.15 & 11.05 & 9.30 & 10.48 & 9.11 & 7.62 & 11.45 & 9.75 & 7.45 & 16.01 & 12.79 & 8.90 \\
\hline VIII & 38.81 & 42.45 & 43.62 & 12.13 & 10.25 & 10.13 & 11.38 & 9.33 & 9.11 & 11.39 & 10.37 & 8.25 & 17.98 & 13.89 & 9.28 \\
\hline IX & 60.70 & 43.30 & 42.06 & 12.76 & 10.13 & 11.89 & 11.12 & 8.82 & 9.95 & 11.94 & 10.31 & 10.10 & 18.48 & 14.04 & 12.93 \\
\hline Mean & 56.51 & 49.65 & 41.60 & 13.09 & 12.56 & 10.83 & 11.67 & 10.46 & 9.38 & 12.40 & 11.49 & 9.72 & 21.74 & 18.74 & 13.80 \\
\hline \multicolumn{16}{|c|}{ Contribution of each character to the divergence } \\
\hline $\begin{array}{l}\text { No. of times } \\
\text { appearing } \\
\text { first in } \\
\text { ranking }\end{array}$ & 25 & 27 & 23 & 0 & 4 & 14 & 1 & 3 & 0 & 0 & 0 & 2 & 47 & 18 & 0 \\
\hline $\begin{array}{c}\% \text { of } \\
\text { contribution } \\
\text { toward total } \\
\text { divergence }\end{array}$ & 0.90 & 0.97 & 0.83 & 0.00 & 0.14 & 0.50 & 0.04 & 0.11 & 0.00 & 0.00 & 0.00 & 0.07 & 1.69 & 0.65 & 0.00 \\
\hline
\end{tabular}




\begin{tabular}{|c|c|c|c|c|c|c|c|c|c|c|c|c|c|c|c|}
\hline \multirow[t]{2}{*}{ Cluster } & \multicolumn{3}{|c|}{100 kernel weight $(g)$} & \multicolumn{3}{|c|}{ Ear weight (g) } & \multicolumn{3}{|c|}{ SCMR } & \multicolumn{3}{|c|}{$\begin{array}{l}\text { Chlorophyll } \\
\text { fluorescence }\end{array}$} & \multicolumn{3}{|c|}{ Leaf firing score } \\
\hline & $\mathrm{N}$ & MS & SS & $\mathrm{N}$ & MS & SS & $\mathrm{N}$ & MS & SS & $\mathrm{N}$ & MS & SS & $\mathrm{N}$ & MS & SS \\
\hline$\overline{\mathbf{I}}$ & 22.22 & 17.80 & 16.33 & 82.36 & 53.07 & 47.64 & 50.19 & 40.15 & 36.71 & 0.69 & 0.64 & 0.61 & 0.58 & 3.93 & 5.03 \\
\hline II & 29.70 & 13.69 & 16.98 & 51.18 & 45.00 & 63.71 & 41.52 & 38.84 & 41.39 & 0.62 & 0.58 & 0.61 & 0.83 & 4.00 & 4.38 \\
\hline III & 21.39 & 20.52 & 19.97 & 90.04 & 75.27 & 81.88 & 47.42 & 44.81 & 43.22 & 0.71 & 0.64 & 0.61 & 0.88 & 3.50 & 4.00 \\
\hline $\mathbf{I V}$ & 27.13 & 20.70 & 12.31 & 139.81 & 95.39 & 23.84 & 54.86 & 48.12 & 33.75 & 0.70 & 0.64 & 0.41 & 0.50 & 3.07 & 7.45 \\
\hline $\mathbf{V}$ & 21.93 & 19.81 & 9.26 & 90.36 & 121.24 & 25.84 & 48.40 & 47.85 & 29.45 & 0.67 & 0.69 & 0.33 & 0.80 & 2.50 & 8.33 \\
\hline VI & 19.95 & 14.61 & 10.34 & 60.23 & 26.60 & 19.48 & 49.58 & 37.19 & 31.14 & 0.66 & 0.43 & 0.57 & 0.83 & 5.91 & 7.46 \\
\hline$\overline{\text { VII }}$ & 20.06 & 12.26 & 9.50 & 41.17 & 19.65 & 15.45 & 44.12 & 31.64 & 28.88 & 0.64 & 0.35 & 0.53 & 1.02 & 6.00 & 8.20 \\
\hline VIII & 21.77 & 13.54 & 10.87 & 54.06 & 23.40 & 20.16 & 43.56 & 34.07 & 27.58 & 0.46 & 0.56 & 0.55 & 1.36 & 5.86 & 7.50 \\
\hline IX & 19.97 & 12.06 & 12.50 & 54.26 & 21.20 & 36.97 & 46.04 & 34.56 & 33.80 & 0.56 & 0.61 & 0.55 & 1.08 & 5.36 & 6.00 \\
\hline Mean & 22.68 & 16.11 & 13.12 & 73.72 & 53.42 & 37.22 & 47.30 & 39.69 & 33.99 & 0.63 & 0.57 & 0.53 & 0.88 & 4.46 & 6.48 \\
\hline \multicolumn{16}{|c|}{ Contribution of each character to the divergence } \\
\hline $\begin{array}{l}\text { No. of times } \\
\text { appearing } \\
\text { first in } \\
\text { ranking }\end{array}$ & 1 & 2 & 13 & 498 & 650 & 1130 & 44 & 45 & 47 & 1271 & 1551 & 1223 & 1 & 0 & 0 \\
\hline $\begin{array}{c}\% \text { of } \\
\text { contribution } \\
\text { toward total } \\
\text { divergence }\end{array}$ & 0.04 & 0.07 & 0.47 & 17.95 & 23.42 & 40.72 & 1.59 & 1.62 & 1.69 & 45.80 & 55.89 & 44.07 & 0.04 & 0 & 0 \\
\hline
\end{tabular}




\begin{tabular}{|c|c|c|c|c|c|c|}
\hline \multirow[t]{2}{*}{ Cluster } & \multicolumn{3}{|c|}{ Harvest index (\%) } & \multicolumn{3}{|c|}{ Grain yield per plant (g) } \\
\hline & $\mathrm{N}$ & MS & SS & $\mathrm{N}$ & MS & SS \\
\hline $\bar{I}$ & 37.80 & 27.22 & 21.80 & 65.43 & 39.03 & 31.17 \\
\hline II & 21.83 & 25.37 & 29.30 & 36.83 & 32.80 & 48.65 \\
\hline III & 39.32 & 35.30 & 36.18 & 70.33 & 57.23 & 61.23 \\
\hline IV & 44.84 & 39.52 & 13.84 & 118.72 & 76.78 & 13.82 \\
\hline $\bar{V}$ & 41.31 & 43.95 & 13.70 & 72.89 & 99.48 & 12.56 \\
\hline VI & 31.15 & 16.97 & 14.81 & 46.04 & 18.18 & 12.61 \\
\hline VII & 23.76 & 13.98 & 11.41 & 29.43 & 12.26 & 9.69 \\
\hline VIII & 32.65 & 15.63 & 11.76 & 40.52 & 14.97 & 11.94 \\
\hline IX & 27.38 & 14.97 & 21.64 & 38.23 & 14.15 & 26.20 \\
\hline Mean & 33.34 & 25.88 & 19.38 & 57.60 & 40.54 & 25.32 \\
\hline \multicolumn{7}{|c|}{ Contribution of each character to the divergence } \\
\hline No. of times appearing first in ranking & 54 & 309 & 79 & 5 & 93 & 25 \\
\hline$\%$ of contribution toward total divergence & 1.95 & 11.14 & 2.85 & 0.18 & 3.35 & 0.90 \\
\hline
\end{tabular}

Note: N- Normal; MS- moderate stress; SS- severe Stress; ASI-Anthesis Silking Interval; SCMR: Spad Chlorophyll Meter Reading 
The genotypes with early pollen shedding and silk emergence and less ASI and least leaf firing score were also included in these clusters indicating that these genotypes were believed to be drought tolerant. Per se performance of these genotypes revealed that these genotypes mature earlier before it coincides with drought and high temperatures during flowering and pollination. As the maize production will be reduced when there is increased ASI due to pollen insufficiency during silk emergence (Hall et al., 1982), or due to pollen dammage under hot dry conditions (Schoper et al., 1986). Keeping in view of this and the cluster pattern the genotypes included in clusters IV and VIII, V and VII and III and V with higher inter cluster distance between them could be involved in crossing programme which would give high manifestation of heterosis with drought tolerance.

\section{References}

AshoftehBeiragi, M., SiahSar, B.A., KhavariKhrasani, S., Golbashy, M., Mahdi Nejad, N. and Ali Zade, A. 2010. Effects of genotype by environment interactions on morphological traits, yield and yield components of new grain corn (Zea mays L.) varieties. Journal of Agroecology. 2(1):136-145.

Banziger, M., Edmeades, G.O., Beck, D. and Bellon, M. 2000. Breeding for drought and nitrogen stress tolerance in maize: From theory to practice, Mexico, D.F. CIMMYT, 68.

Bassetti, P. and Westgate, M. E. 1993. Senescence and receptivity of maize silks. Crop Science. 33: 275-278.

Chen FaBo, Yang KeCheng, Rong Ting Zhao and Pan GuangTang. 2007. Analysis of genetic diversity of maize hybrids in the regional tests of Sichuan and Southwest China. Acta Agronomica Sinica. 33(6): 991-998.
Denby, K. and Gehring, C. 2005. Engineering drought and salinity tolerance in plants: lessons from genome-wide expression profiling in Arabidopsis. Trends Biotech. 23:547-552.

Hall, A. J., Vilella, N., Trapani, N. and Chimenti, C. 1982. The effects of water stress and genotype on dynamics of pollen shedding and silking in maize. Field Crops Research. 5:349-363.

Khan, I.A., Habib, S., Sadaqat, H.A. and Tahir, M.N.H. 2004. Selection criteria based on seedling growth parameters in maize varies under normal and water stress conditions. International Journal of Agriculture and Biology. 6(2):252256.

Mahalanobis, P. C.1936. On the generalized distance in statistics. Proceedings National Institute of Science. India. 2(1): 49-55.

Murthy, B. R. and Arunachalam, V. 1966. The nature of divergence in relation to breeding system in crop plants. Indian Journal of Genetics. 26A: 188-198.

NeSmith, D. S. and Ritchie, J. T. 1992. Effects of soil water deficits during tassel emergence on development and yield components of maize (Zea mays L.). Field Crops Research. 28: 251-256.

Rao, C. R. 1952. Advanced Statistical Methods in Biometrical Research. John Wiley and Sons, New York. 390.

Saxena, V. K., Mathi, N. S., Singh, N. N. and Vasal, S. K. 1998. Heterosis in maize: Grouping and patterns. Proc. of 7th Asian Regional Maize Workshop. Los Banos, Philippines. February 23-27, 124-133.

Schoper, J. B., Lambert, R. J. and Vailas, B. L., 1986. Maize pollen viability and ear receptivity under water and high temperature stress. Crop Science. 26:1029-1033.

Umar, U. U., Ado, S.G., Aba, D.A. and Bugaje, S.M. 2015. Studies on genetic 
variability in maize (Zea mays, L.) under stress and non-stress environmental conditions. International Journal of Agronomy and Agricultural Research. 7(1): 70-77.
Vasal, S. K. 1998. Hybrid maize technology: Challenges and expanding possibilities for research in the next century. Proc. of 7th Asian Regional Maize Workshop. Los Banos, Philippines. February 23-27, 58-62.

\section{How to cite this article:}

Vijay Kumar K., K. V. Radha Krishna, T. Pradeep, D. Vishnuvardhan Reddy and Bhave M. H. V. 2018. Genetic Divergence of Maize Genotypes under Normal and Water Stress Conditions. Int.J.Curr.Microbiol.App.Sci. 7(06): 1441-1451. doi: https://doi.org/10.20546/ijcmas.2018.706.171 